\title{
PRECISIÓN DE LA TAQUIPNEA Y LAS RETRACCIONES SUBCOSTALES COMO SIGNOS CLÍNICOS PARA DIAGNÓSTICO DE NEUMONÍA ADQUIRIDA EN LA COMUNIDAD EN NIÑOS: REVISIÓN SISTEMÁTICA Y METAANÁLISIS
}

\author{
Juan Pablo Domecq, ${ }^{1,2, a}$, Gabriela Prutsky1,2,a, María de los Ángeles Lazo, , Carlos Salazar, ${ }^{1, b}$, \\ Víctor Montori ${ }^{1,2, c}$, Yolanda Prevost ${ }^{1, d}$, Luis Huicho ${ }^{3,4, e}$, Patricia Erwin ${ }^{2, f}$, Germán Málaga ${ }^{1,9}$
}

RESUMEN

Objetivos. Evaluar si la taquipnea y las retracciones subcostales son predictoras eficientes para el diagnóstico de neumonía adquirida en la comunidad (NAC) en niños. Materiales y métodos. Se revisaron las bases de datos: PubMed, LILACS, The African Journal Database y The Cochrane Central Library. Se incluyeron estudios originales que evaluaron el rendimiento diagnóstico de los criterios clínicos de taquipnea o retracciones subcostales, definidos según los criterios de la Organización Mundial de la Salud (OMS) para el diagnóstico de NAC en niños de hasta cinco años de edad con tos y fiebre. Se estimaron las razones de probabilidades (LR), el odds ratio diagnostico (DOR) y sus respectivos intervalos de confianza al 95\% (IC95\%) para cada prueba clínica evaluada. Resultados. Se encontraron 975 estudios, incluyendo ocho en la revisión. Se enrolaron 4740 pacientes y analizaron 3584 (75\%), de los cuales 916 (19\%) tuvieron el diagnóstico de NAC. Al combinar los datos, la taquipnea obtuvo una LR positiva de 3,16, (IC95\% 2,11-4,73) y una LR negativa de 0,36 (IC95\% 0,23-0,57). El DOR fue de 10,63 (IC95\% 4,4-25,66, $\left.I^{2}=93 \%\right)$. Al realizar el análisis para retracciones subcostales se obtuvo un LR positivo de $2,49($ IC95\% 1,41-4,37) y un LR negativo de 0,59 (IC95\% 0,4-0,87). EI DOR fue de 5,32 (IC95\% 1,88-15,05, I²=89\%). Conclusiones. Se puede tomar en cuenta la presencia o ausencia de taquipnea y retracciones subcostales en el diagnóstico de NAC, cabe considerar la incertidumbre relativa en su poder diagnóstico y los LR relativamente modestos. La confianza en estos resultados es baja por la inadecuada calidad de la evidencia en este tema.

Palabras clave: Frecuencia respiratoria; Neumonía; Niños; Metanálisis; Revisión sistemática (fuente: DeCS BIREME).

\section{IDENTIFICATION OF TACHYPNEA AND SUBCOSTAL RETRACTIONS AS CLINICAL SIGNS FOR THE DIAGNOSIS OF COMMUNITY-ACQUIRED PNEUMONIA AMONG CHILDREN: SYSTEMATIC REVIEW AND META-ANALYSIS}

\section{ABSTRACT}

\begin{abstract}
Objectives. Determine whether tachypnea and subcostal retractions can be efficient predictors for the diagnosis of Community-Acquired Pneumonia (CAP) among children. Materials and methods. These were the databases used: PubMed, LILACS, The African Journal Database and The Cochrane Central Library. Original studies were included which assessed the diagnostic performance of the clinical criteria for tachypnea or subcostal retraction defined in accordance with the criteria of the World Health Organization (OMS) for CAP diagnosis in children $\leq 5$ with cough and fever. The likelihood ratio (LR), the diagnosis odds ratio (DOR), and their respective confidence intervals at 95\% (IC95\%) were estimated for each clinical test. Results. 975 studies were found, eight were included in the review. 4740 patients were enrolled and 3584 (75\%) were analyzed, $916(19 \%)$ of which had a CAP diagnosis. When data were combined, tachypnea had a positive LR of 3.16, $(95 \% \mathrm{Cl} 2.11-4.73)$ and a negative LR of 0.36 (95\% Cl 0.23-0.57). The DOR was 10.63 (95\% Cl 4.4-25.66, I2=93\%). When subcostal retractions were analyzed, a positive LR of $2.49(95 \% \mathrm{Cl} 1.41-4.37)$ and a negative LR of $0.59(95 \% \mathrm{Cl} 0.4-0.87)$ were obtained. The DOR was $5.32(95 \% \mathrm{Cl} 1.88-15.05$, I2=89\%). Conclusions. The presence or absence of tachypnea and subcostal retractions can be used in CAP diagnosis; it is worth considering the relative uncertainty in its diagnostic power and relatively modest LR. The confidence of these results is low due to the inadequate quality of the related evidence.
\end{abstract}

Key words: Respiratory rate; Pneumonia; Child; Meta-analysis; Review, systematic (source: MeSH NLM).

\footnotetext{
Unidad de Conocimiento y Evidencia, Universidad Peruana Cayetano Heredia. Lima, Perú.

Knowledge and Evaluation Research Unit, Mayo Clinic. Minnesota, EE.UU.

Facultad de Medicina "Alberto Hurtado", Universidad Peruana Cayetano Heredia. Lima, Perú.

Facultad de Medicina Humana, Universidad Nacional Mayor de San Marcos. Lima, Perú.

Médico cirujano, ${ }^{\mathrm{b}}$ médico internista, ${ }^{\mathrm{c}}$ médico endocrinólogo, ${ }^{\mathrm{d}}$ médico pediatra neonatólogo, ${ }^{\mathrm{e}}$ médico pediatra, ${ }^{\mathrm{f}}$ bibliotecóloga magister en ciencias bibliotecarias, ${ }^{\mathrm{g}}$ médico magister en Medicina

Recibido: 13-06-12 Aprobado: 29-08-12
}

Citar como: Domecq JP, Prutsky G, Lazo MA, Salazar C, Montori V, Prevost Y, et al. Precisión de la taquipnea y las retracciones subcostales como signos clínicos para diagnóstico de neumonía adquirida en la comunidad en niños: revisión sistemática y metaanálisis. Rev Peru Med Exp Salud Publica. 2012;29(3):337-44 


\section{INTRODUCCIÓN}

La neumonía adquirida en la comunidad (NAC) es un proceso inflamatorio agudo del parénquima pulmonar en individuos que no han sido hospitalizados recientemente ${ }^{(1)}$. Es una de las principales causas de morbimortalidad en niños. La Organización Mundial de la Salud (OMS) estima que de los 156 millones de casos por año, en niños menores de cinco años, 20 millones requieren hospitalización y hasta 2 millones mueren de NAC; por tanto, esta mortalidad es mayor que las muertes causadas por HIVISIDA, malaria y tuberculosis combinadas ${ }^{(2)}$. Por esta razón, la NAC se ha convertido en la principal causa de muerte en niños menores de 5 años a nivel mundial.

La NAC es más prevalente y severa en países de bajos ingresos. El 97\% (152 millones por año) de los niños menores de cinco años con neumonía viven en estos países ${ }^{(3)}$ en donde enfrentan 17 veces más riesgo de morir que los niños con NAC en países de altos ingresos ${ }^{(4)}$. La NAC infantil persiste como uno de los problemas de salud pública más importantes que afrontan países como el Perú.

El tratamiento antibiótico temprano administrado en la comunidad se asocia con mejores desenlaces en cuanto a morbimortalidad ${ }^{(5,6)}$. Sin embargo, como señalan Marsh et al. (7), en zonas rurales, la implementación de estas estrategias se ve limitada por la escasez de personal que sea capaz de reconocer la NAC. Con el fin de mejorar el diagnóstico de la NAC, la OMS estableció en 1991 criterios basados en signos clínicos con suficiente capacidad diagnóstica, y útiles en manos de personal sanitario con mínimo entrenamiento, permitiendo así el uso adecuado de antibióticos en zonas rurales ${ }^{\left({ }^{8}\right)}$.

La decisión de la OMS se originó a partir de un estudio realizado en Filadelfia, Estados Unidos ${ }^{(9)}$, en el que se revisaron 29 signos clínicos, concluyendo que "respiración rápida" es un mejor predictor de neumonía que los hallazgos auscultatorios. Estudios adicionales permitieron establecer los puntos de corte de 50 y 40 respiraciones por minuto en lactantes entre 2-11 meses y niños entre 1 y 5 años, respectivamente ${ }^{(8)}$. Con respecto a las retracciones subcostales, se pudo verificar su asociación con neumonía severa ${ }^{(10)}$. Posteriormente, estos datos fueron corroborados en estudios de campo, sin considerar la posible sobreestimación de la capacidad diagnóstica que los diseños de esos estudios pudiesen causar ${ }^{(5,11)}$.

Luego de más de 20 años de implementada esta estrategia, su eficacia se apoya en estudios observacionales de campo, con alto riesgo de sesgo. Al ser estudios observacionales, la utilización de cointervenciones, como por ejemplo: educación sobre reconocimiento temprano de las manifestaciones clínicas de la enfermedad; visita domiciliaria activa por parte de los prestadores de salud, o el estado de inmunización previo de los participantes, no fueron equitativamente balanceadas entre los grupos $y$, también, los resultados no fueron ajustados considerando estas variables. Además, en la mayoría de los casos, los grupos control no tenían acceso a antibióticos, creando así un desbalance de riesgos que afectaba directamente la morbimortalidad de los grupos evaluados ${ }^{(5)}$. Estas limitaciones sumadas a que la disminución de la tasa de mortalidad infantil por cualquier causa no ha sido acompañada de una disminución en la tasa de mortalidad por NAC, generan sospechas sobre la real efectividad de este estrategia ${ }^{(11)}$.

La presente revisión sistemática pretende evaluar si los signos clínicos, taquipnea y retracciones subcostales, definidos según los criterios de la OMS pueden ser predictores eficientes de NAC en niños, los resultados permitirían un manejo antibiótico inicial racional en niños que presenten fiebre y tos.

\section{MATERIALES Y MÉTODOS}

\section{CRITERIOS DE ELEGIBILIDAD}

Se incluyeron estudios observacionales tipo cohortes prospectivos o retrospectivos que ayudarán a evaluar el rendimiento diagnóstico de la taquipnea o las retracciones subcostales, definidos por la OMS para el inicio de tratamiento antibiótico en niños de cinco años o menores, con sospecha diagnóstica de neumonía (tos y fiebre). Los estudios debían de tener una prueba de oro para el diagnóstico de neumonía (definido por los autores de cada estudio original). Los estudios con diseño caso y control (enfermos frente a sanos) sin incertidumbre diagnóstica, fueron excluidos debido a que no representan el escenario real, en donde los clínicos van a aplicar los resultados del estudio.

Además, se excluyeron estudios en pacientes severamente malnutridos, con acidosis metabólica, deshidratación u otras comorbilidades, incluyendo síndrome de Down, insuficiencia cardiaca, malformación broncopulmonar, fibrosis quística, tuberculosis, entre otras. También fueron excluidos artículos con información insuficiente para obtener una tabla de contingencia (tabla 2x2), después de haber contactado a los autores y no haber obtenido respuesta, en un plazo prudente (dos correos electrónicos sin respuesta en un lapso de dos semanas). 


\section{ESTRATEGIA DE BÚSQUEDA}

Con ayuda de una bibliotecaria experta (PE) fueron exploradas las siguientes bases de datos: PubMed (MedLine), LILACS (base de datos de Latinoamérica y el Caribe), The African Journal database y The Cochrane Central Library. La búsqueda abarcó desde la fecha de inserción de cada base de datos hasta febrero de 2012.

Se realizó una búsqueda de alta sensibilidad en cada una de las bases de datos biomédicas listadas anteriormente, para tratar de identificar todos los posibles estudios relevantes. Elaboramos un filtro de búsqueda utilizando una combinación de términos MeSH (Medical Subject Heading) como: "acute respiratory infection", "pneumonia", "tachypnea", "difficult breathing", "chest retraction", "chest indrawing", combinados con términos boleanos "OR" y "AND" (ver Anexo).

La búsqueda electrónica fue complementada con una búsqueda manual de estudios originales elegibles que consistió en revisar las listas bibliográficas de revisiones sistemáticas y narrativas con similares preguntas de investigación. Dos de los autores, expertos en neumonía pediátrica (YP y LH) buscaron literatura en fuentes no indexadas (biblioteca de la Universidad Peruana Cayetano Heredia y la biblioteca del Instituto de Salud del Niño (INSN) de Perú). Además, contactaron otros expertos en el tema en sus respectivos centros de atención (Hospital Nacional Cayetano Heredia e INSN) solicitando información respecto de potenciales estudios elegibles que no hayan sido identificados por nuestra estrategia de búsqueda. La búsqueda manual no identificó estudios adicionales.

\section{SELECCIÓN DE LOS ESTUDIOS Y EXTRACCIÓN DE DATOS}

Dos de los autores (YP y GM) aplicaron los criterios de elegibilidad para evaluar las referencias de los artículos obtenidos por nuestra estrategia de búsqueda, examinando el título y el resumen. Este proceso se realizó por duplicado y de forma independiente. Posteriormente, se obtuvieron los artículos completos de las referencias potencialmente elegibles, y de manera independiente y en duplicado, cuatro autores (JPD, CS, GP y GM) realizaron el tamizaje de las mismas, decidiendo la inclusión de estas en la revisión. Los mismos autores realizaron la extracción de datos de interés y evaluaron la calidad metodológica de los estudios incluidos. Las discrepancias fueron discutidas y resueltas por consenso.

\section{CALIDAD METODOLÓGICA}

Evaluamos la calidad metodológica utilizando los criterios de QUADAS (Quality Assessment of Diagnostic
Accuracy Studies) ${ }^{(12)}$. Además, el grupo de investigación creyó conveniente añadir dos preguntas para evaluar la calidad de los estudios:

- ¿Los evaluadores del estándar de oro fueron los adecuados o presentaban el entrenamiento necesario para realizar la prueba?

- ¿Los evaluadores de la prueba a evaluar fueron los adecuados o presentaban el entrenamiento necesario para realizar la prueba?

El grupo de investigación decidió incluir estas dos preguntas debido a que la calidad y capacidad de los evaluadores de la prueba de interés y la del estándar de oro estarían directamente relacionadas con los resultados, además de limitar la aplicabilidad de estos. Por ejemplo, los evaluadores podrían estar sobreentrenados para detectar los signos clínicos o inexpertos en el uso del estándar de oro.

\section{ANÁLISIS ESTADÍSTICO}

El grado de concordancia interobservador más allá de lo que podría atribuirse al azar (coeficiente kappa), fue calculado para el tamizaje de textos completos y en la evaluación de la calidad metodológica de los estudios incluidos.

Se utilizó el programa Meta-Disc 1.4 para metaanálisis de pruebas de tamizaje y diagnósticas ${ }^{(13)}$. Empleando el método de efectos aleatorios, combinamos los valores de sensibilidad, especificidad, razón de probabilidades (LR) y odds ratios diagnósticos (DOR) de cada estudio primario, y estimamos el intervalo de confianza al 95\% alrededor de cada uno de estos resultados combinados. Debido a que los valores de sensibilidad y especificidad son valores interconectados (es decir, cambian juntos en dirección opuesta cuando varían los criterios de positividad de una prueba), decidimos basar el análisis primario en los estimados de LR y DOR. LR es la razón de la probabilidad de un resultado de la prueba diagnóstica (positiva $\mathrm{O}$ negativa) en pacientes con NAC sobre la probabilidad de ese mismo resultado (positivo o negativo) en pacientes sin NAC. Estos LR pueden ser multiplicados por la probabilidad preprueba de presentar NAC (estimados de prevalencia de NAC en la población pertinente) para así obtener los odds posprueba. Los LR cuantifican el poder del resultado para cambiar la probabilidad preprueba a la probabilidad posprueba. Un LR de 1 indica una prueba inútil; un LR mayor a 10 o menor de 0,1 indica que la prueba es de gran utilidad diagnóstica. EI DOR es la razón de odds de estar enfermo y tener una prueba positiva frente al odds de estar enfermo y tener una prueba negativa, y es indicador útil para la evaluación de inconsistencias entre los estudios y resume el rendimiento de la prueba ${ }^{(14)}$. 


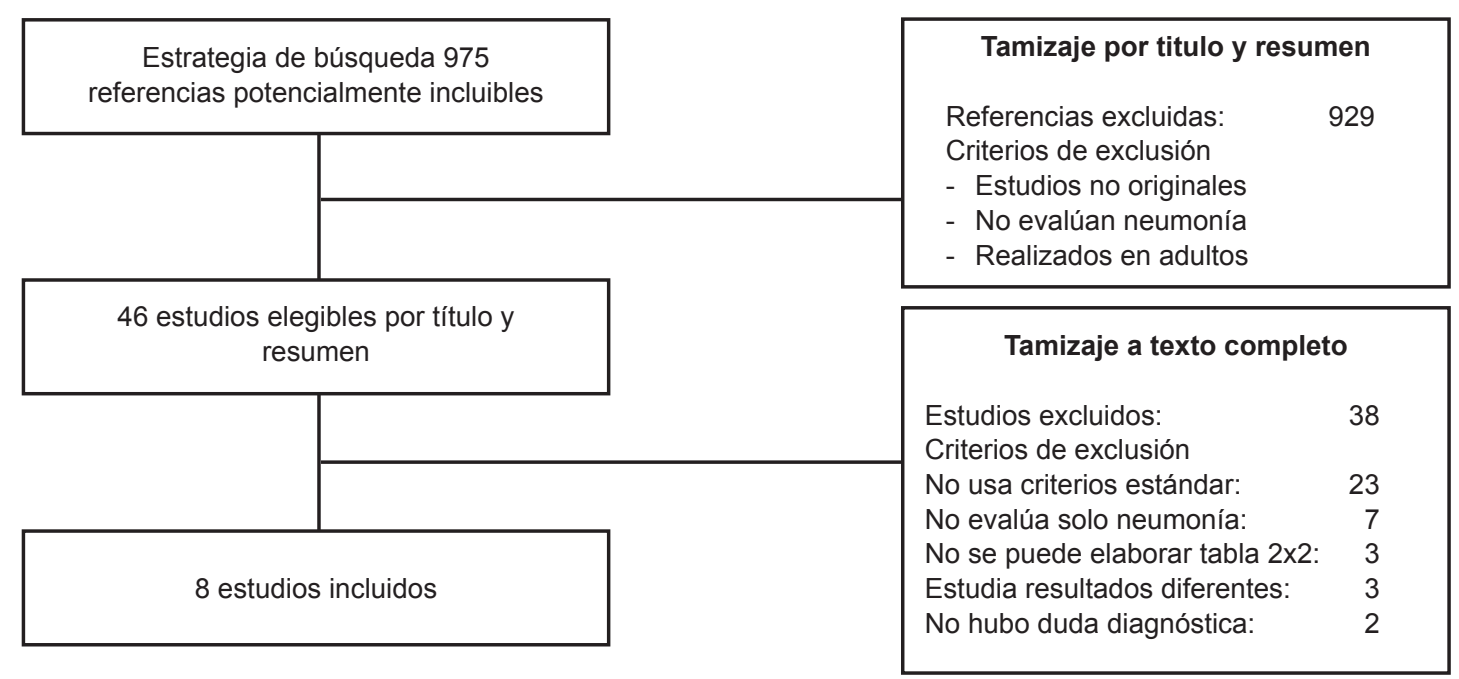

Figura 1. Proceso de selección de los artículos incluidos en el estudio.

Se utilizó la prueba de chi ${ }^{2}$ para evaluar la heterogeneidad de los resultados, y la prueba de $I^{2}$ para cuantificar el grado de inconsistencias no explicables por el azar entre los resultados de los estudios incluidos ${ }^{(15)}$.

Para explicar posibles inconsistencias, por protocolo planeamos evaluar interacción entre la capacidad diagnóstica y los siguientes subgrupos:

- Calidad metodológica de los estudios incluidos, considerando alto grado de sesgo de selección frente a moderado o bajo riesgo de sesgo de selección.

- Nivel de atención (primer nivel de atención frente a otro nivel de atención).
- Países con bajos ingresos frente a altos ingresos. Utilizando la clasificación del banco mundial de acuerdo al ingreso nacional bruto per cápita ${ }^{(16)}$.

- Prevalencia de neumonía en los estudios incluidos analizado en metarregresión.

\section{RESULTADOS}

\section{RESULTADOS DE LA BÚSQUEDA}

Se encontraron 975 estudios, de los que se eligieron 46 por título y resumen. Finalmente, ocho fueron incluidos en la revisión ${ }^{(10,17-24)}$ (Figura 1). El grado de concordancia interobservador fue casi perfecto $(\mathrm{k}=0,92)^{(25)}$.

Tabla 1. Características de los estudios incluidos.

\begin{tabular}{|c|c|c|c|c|c|c|}
\hline $\begin{array}{l}\text { Autor } \\
\text { Año }\end{array}$ & $\begin{array}{l}\text { Diseño } \\
\text { de estudio }\end{array}$ & Criterios de elegibilidad & País & Lugar & Población & Edad \\
\hline $\begin{array}{l}\text { DAl } \\
1995 \text { (17) }\end{array}$ & $\begin{array}{l}\text { Cohorte } \\
\text { prospectiva }\end{array}$ & $\begin{array}{l}\text { Todo niño entre } 2 m \text { y } 5 a \text { con tos que no haya recibido } \\
\text { antibióticos en las últimas } 4 \text { semanas }\end{array}$ & China & Hospitalario & 701 & $\begin{array}{l}2 \text { meses - } \\
5 \text { años }\end{array}$ \\
\hline $\begin{array}{l}\text { FALADE } \\
1995^{(18)}\end{array}$ & $\begin{array}{l}\text { Cohorte } \\
\text { prospectiva }\end{array}$ & $\begin{array}{l}\text { Todo niño que acudió a la emergencia por tos y } \\
\text { dificultad respiratoria. }\end{array}$ & Gambia & Hospitalario & 255 & $\begin{array}{l}3 \text { meses - } \\
5 \text { años }\end{array}$ \\
\hline $\begin{array}{l}\text { HARARI } \\
1991^{(19)}\end{array}$ & $\begin{array}{l}\text { Cohorte } \\
\text { prospectiva }\end{array}$ & Pacientes que tenían tos, entre 8 semanas y 6 años. & $\begin{array}{l}\text { Papua } \\
\text { Guinea }\end{array}$ & $\begin{array}{l}\text { Hospitalario } \\
\text { (pediatría) }\end{array}$ & 185 & $\begin{array}{l}8 \text { sem. - } \\
5 \text { años }\end{array}$ \\
\hline $\begin{array}{l}\text { PALAFOX } \\
2000^{(20)}\end{array}$ & $\begin{array}{l}\text { Cohorte } \\
\text { prospectivo }\end{array}$ & $\begin{array}{l}\text { Niños con sospecha clínica de neumonía que } \\
\text { requirieron atención medica con un tiempo de } \\
\text { enfermedad menor a } 2 \text { semanas. }\end{array}$ & México & $\begin{array}{l}\text { Hospital de } \\
\text { referencia }\end{array}$ & 110 & $\begin{array}{l}3 \text { días - } \\
5 \text { años }\end{array}$ \\
\hline $\begin{array}{l}\text { RED } \\
1992\end{array}$ & $\begin{array}{l}\text { Cohorte } \\
\text { prospectiva }\end{array}$ & $\begin{array}{l}\text { Niños que se presentaron al servicio de consulta } \\
\text { externa entre las } 7 \text { y } 14 \mathrm{~h} \text { de lunes a sábado. Se } \\
\text { enroló a cada quinto niño traído por tos o fiebre. }\end{array}$ & Malawi & Hospitalario & 1469 & $\leq 5$ años \\
\hline $\begin{array}{l}\text { SHAMO'ON } \\
2004^{(22)}\end{array}$ & $\begin{array}{l}\text { Cohorte } \\
\text { prospectiva }\end{array}$ & $\begin{array}{l}\text { Pacientes ambulatorios provenientes de una clínica a } \\
20 \mathrm{~km} \text { del hospital y que se hospitalizaron en el hospital } \\
\text { por que cumplían los criterios de la WHO. }\end{array}$ & Jordania & Hospitalario & 147 & $\leq 5$ años \\
\hline $\begin{array}{l}\text { SHAH } \\
2009 \text { (23) }\end{array}$ & $\begin{array}{l}\text { Cohorte } \\
\text { retrospectiva }\end{array}$ & $\begin{array}{l}\text { Pacientes atendidos en la emergencia del hospital } \\
\text { con sospecha de neumonía enviados a tomar una } \\
\text { radiografía de tórax. }\end{array}$ & $\begin{array}{l}\text { Estados } \\
\text { Unidos }\end{array}$ & $\begin{array}{l}\text { Hospitalario, } \\
\text { emergencia } \\
\text { pediátrica }\end{array}$ & 1622 & $\leq 5$ años \\
\hline $\begin{array}{l}\text { SINGHI } \\
1994(24)\end{array}$ & $\begin{array}{l}\text { Cohorte } \\
\text { prospectiva }\end{array}$ & $\begin{array}{l}\text { Pacientes atendidos por emergencia por historia de } \\
\text { fiebre con o sin tos. }\end{array}$ & India & Hospitalario & 251 & $\leq 2$ meses \\
\hline
\end{tabular}




\section{CARACTERÍSTICAS DE LOS ESTUDIOS}

Todos los estudios incluidos evaluaron taquipnea, y seis estudios evaluaron retracciones subcostales de acuerdo con la definición dada por la OMS (Tabla 1). En total, estos estudios enrolaron 4740 pacientes y analizaron 3584 (75\%), de los cuales 916 (19\%) tuvieron el diagnóstico de NAC.

\section{CALIDAD METODOLÓGICA}

Todos los estudios incluidos utilizaron como comparación un estándar de oro definido por cada uno de los autores. Los evaluadores de la prueba de oro fueron considerados como adecuados en cada uno de los estudios. La prueba de oro en todos los estudios fue la radiografía de tórax (aunque la evaluación fue variable o no descrita). Ambas pruebas (prueba de oro frente a la prueba a evaluar) fueron interpretadas de manera independiente (cegadas a los resultados de la otra prueba) y el $75 \%$ de los estudios aplicó la prueba de oro a toda la población o a una muestra representativa de esta, seleccionada al azar.

El $80 \%$ de los estudios incluidos no describió detalladamente el estándar de oro y el $50 \%$ no lo hizo con la prueba a evaluar, limitando así su reproducibilidad o validez externa (ver Anexos). El 75\% de los estudios incluidos no presentó los resultados indeterminados en el estándar de oro o en la prueba a evaluar, puesto que estos resultados podrían haber sido excluidos del análisis, existiendo un alto riesgo de sesgo de selección de la muestra. El $38 \%$ de los estudios incluidos informó pérdidas durante el seguimiento sin detallar las circunstancias o motivos. El $75 \%$ de los estudios no informó el tiempo entre la realización de la prueba a evaluar y el estándar de oro, por lo que la posibilidad de que estas pruebas estuvieran midiendo diferentes espectros de NAC o diferentes enfermedades debido a nuevos eventos, complicaciones, enfermedades sobreagregadas, recuperación espontánea o progresión, es alta. El grado de concordancia interobservador fue sustancial >0,70 (25).

\section{HALLAZGOS}

Taquipnea como signo clínico para el diagnóstico de NAC: el LR combinado en presencia de taquipnea es 3,16 (IC95\% 2,11-4,73, $I^{2}=93$ ) y el LR combinado en ausencia de taquipnea es 0,36 (IC95\% 0,23-0,57, $\left.\right|^{2}=95$ ), Figura 2A. El DOR para taquipnea es 10,64 (IC95\% $\left.4,41-25,67, I^{2}=94 \%\right)$, Figura $3 A$.

Retracciones subcostales como signo clínico para el diagnóstico de NAC: el LR combinado para presencia

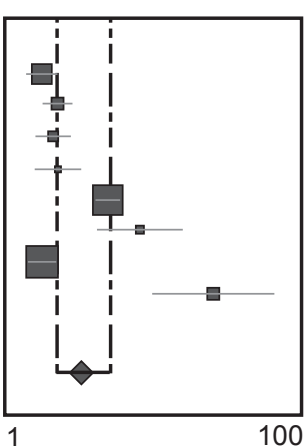

$\begin{array}{lrc} & \text { LR positivo (IC 95\%) } \\ \text { DAI } & 1,71 & (1,35-2,17) \\ \text { FALADE } & 2,24 & (1,78-2,82) \\ \text { HARARI } & 2,01 & (1,52-2,65) \\ \text { PALAFOX } & 2,23 & (1,53-3,24) \\ \text { RED } & 4,88 & (4,02-5,93) \\ \text { SHAMOON } & 8,05 & (4,02-16,12) \\ \text { SHAH } & 1,70 & (1,32-2,19) \\ \text { SHINGUI } & 26,13 & (9,97-69,57)\end{array}$

Efecto aleatorio:

LR Positivo $\quad 3,16(2,11-4,73)$ $1^{2}=93,1 \%$

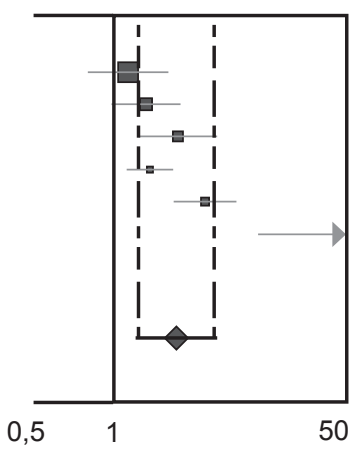

\section{A. Taquipnea}

Peso
14,3
13,5
13,0
11,7
14,1
10,4
8,8
14,3

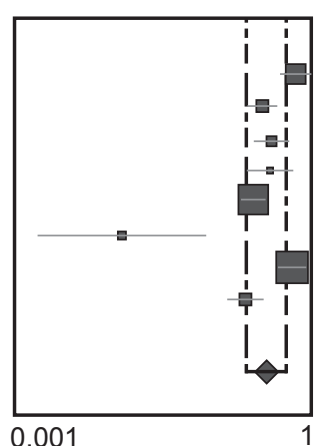

\section{B. Retracciones}

$\begin{array}{lrlr} & \text { LR positivo (IC 95\%) } & \text { Peso } \\ \text { DAI } & 1,25 & (0,70-2,25) & 19,0 \\ \text { FALADE } & 1,57 & (0,95-2,60) & 18,9 \\ \text { HARARI } & 2,59 & (1,43-4,70) & 17,8 \\ \text { PALAFOX } & 1,73 & (1,23-2,43) & 18,7 \\ \text { SHAMOON } & 3,85 & (2,37-6,24) & 18,6 \\ \text { SHINGUI } & 63,34 & (8,88-451,98) & 6,9 \\ \text { Efecto aleatorio: } & \\ \text { LR Positivo } & 2,49 \quad(1,42-4,37) \\ \text { I }^{2}=83,1 \% & \end{array}$

$\begin{array}{lccc} & \text { LR negativo (IC 95\%) } & \text { Peso } \\ \text { DAI } & 0,98 & (0,93-1,03) & 20,6 \\ \text { FALADE } & 0,88 & (0,75-1,03) & 18,2 \\ \text { HARARI } & 0,77 & (0,64-0,94) & 17,3 \\ \text { PALAFOX } & 0,49 & (0,28-0,85) & 12,5 \\ \text { SHAMOON } & 0,16 & (0,09-0,28) & 13,3 \\ \text { SHINGUI } & 0,54 & (0,45-0,65) & 18,2 \\ & & & \\ & & \\ \text { Efecto aleatorio: } & & \\ \text { LR Negativo } & 0,59 & (0,40-0,87) \\ \text { I }^{2}=96,4 \% & & \end{array}$

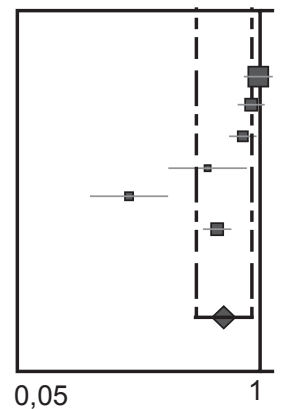

Figura 2. Metaanálisis de razón de probabilidades (LR). A. Taquipnea, B. Retracciones subcostales. Nota. El peso corresponde al aporte de cada estudio en el análisis. 


\section{A. Taquipnea}

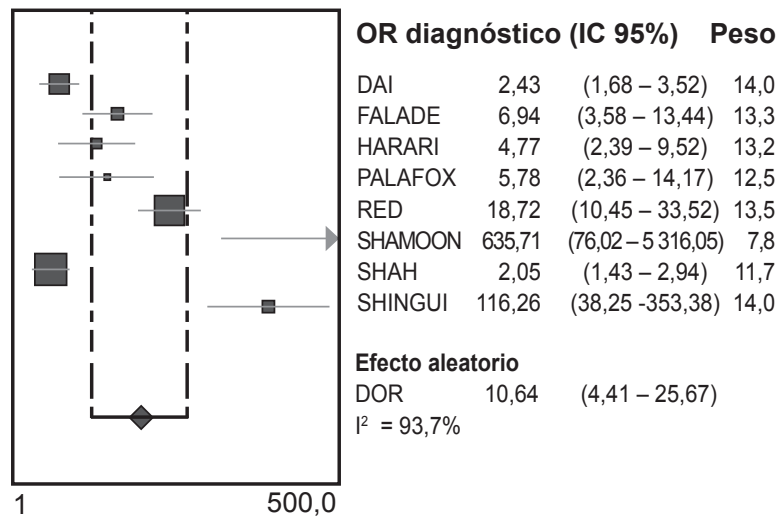

\section{B. Retracciones}

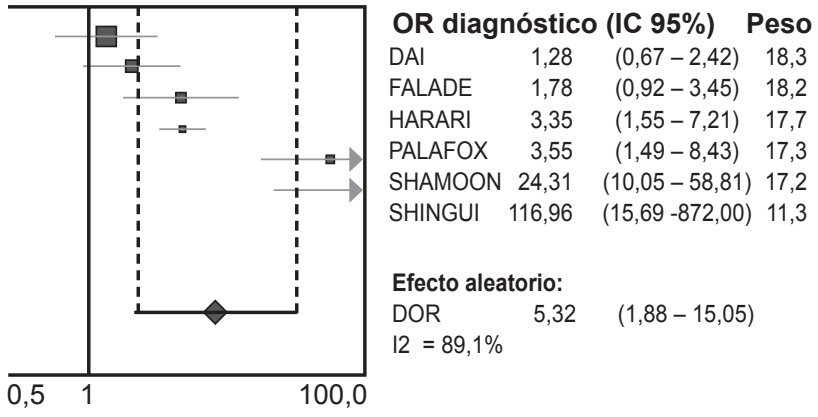

Figura 3. Metanálisis de Odds Ratio Diagnóstico (DOR). A. Taquipnea, B. Retracciones subcostales. Nota. El peso corresponde al aporte de cada estudio en el análisis.

de retracciones es 2,49 (IC95\% $\left.1,42-4,37, I^{2}=83\right)$ y para ausencia de retracciones 0,59 (IC95\% 0,40-0,87, $\left.\right|^{2}=96$ ), Figura 2B. El DOR combinado para retracciones es 5,32 (IC95\% 1,88-15,05, I'2=89\%), Figura 3B.

En los anexos se resumen los LR combinados para las dos pruebas clínicas evaluadas, estén estas presentes (LR positivo) o ausentes (LR negativo). Los clínicos pueden utilizar este nomograma para evaluar la probabilidad de que sus pacientes presenten NAC. La probabilidad preprueba indicada en el gráfico se debe generar de la prevalencia de la enfermedad en la población de donde proviene el paciente y la sospecha clínica derivada de la anamnesis.

No contamos con un número suficiente de estudios como para poder realizar los análisis por subgrupos planteados en el protocolo.

\section{DISCUSIÓN}

La información respecto al uso eficiente de signos clínicos es muy importante para los clínicos, en especial en medios donde el acceso a recursos de salud, incluyendo la tecnología y pruebas diagnósticas, es limitado o inexistente. La evidencia existente indica que tanto taquipnea como retracciones subcostales son moderadamente útiles como pruebas diagnósticas para aumentar la probabilidad de enfermedad cuando están presentes, pero no son suficientemente útiles para disminuir la probabilidad de NAC. Ninguna de las dos es suficiente por sí sola para confirmar o descartar neumonía. Los estudios que generaron esta evidencia carecen de algunas protecciones metodológicas lo que quizás contribuyó a sobrestimar su rendimiento/ desempeño.

La heterogeneidad podría deberse a que las habilidades reales de los evaluadores difirió entre los estudios incluidos, aun cuando fueron todos informados $y$ categorizados como adecuados. Además, los criterios de selección de los pacientes, como se muestra en la Tabla 2, son muy amplios pudiendo presentar sesgos de selección no presentados. Esto podría explicar la gran sensibilidad que presenta Shamoon $2004{ }^{(22)}$, cuyos pacientes fueron enrolados en un hospital de referencia con un espectro de enfermedad más severo $\mathrm{y}$, probablemente, con mayor bagaje sintomático que aquellos que fueron evaluados en centros comunitarios. En otras palabras, el proceso de referencia y transferencia al hospital podría haber seleccionado los casos más graves o los casos persistentes, mientras que los leves o con resolución espontánea no habrían acudido al hospital, incrementando así la sensibilidad y especificidad de la prueba ${ }^{(26)}$. Además, la alta prevalencia de neumonía que muestran los estudios incluidos sugiere cierto sesgo de selección o referencia. Un aspecto adicional que debe ser considerado, es el hecho de que la OMS, recomienda el uso de retracciones subcostales para la identificación de neumonía severa mientras que en los estudios incluidos este signo ha sido utilizado como parte del diagnóstico de neumonía en general, lo cual creemos disminuye el poder diagnóstico de este signo.

Una revisión sistemática publicada en el 2005 encontró que la ausencia de síntomas y signos respiratorios, como rinorrea, tos, retracciones, taquipnea, murmullo vesicular disminuido, aleteo nasal, y quejido espiratorio, descartaban el diagnóstico de neumonía con un LR negativo de 0,0 (IC 95\% 0,0-0,4), mientras que la presencia de al menos uno de estos presentaba un LR positivo de 1,6 (IC95\% 1,3-31) (26). Estos hallazgos pueden ser aplicados solo en países desarrollados por su baja prevalencia de NAC (27). En países como Perú, en donde la prevalencia de NAC es más elevada, la presencia de al menos uno de los signos 
listados anteriormente incrementaría las sospechas diagnósticas de los médicos tratantes y justificaría la utilización de otros procedimientos diagnósticos como la radiografía de tórax o la administración temprana de antibióticos.

Los estudios realizados por Harari et al. (19) y Zukin et al. (28) que evaluaron la fiebre como predictor de NAC, mostraron que esta carece de utilidad para confirmar o descartar esta enfermedad, encontrando LR positivos entre 1,1 a 1,5 y LR negativos entre 0,17 a 0,95.

Con base en la información presentada, no existe evidencia suficiente para desestimar el valor de los signos clínicos recomendados por la OMS para el diagnóstico de neumonía y tratamiento antibiótico temprano ${ }^{(29)}$. No obstante, debemos tener en cuenta que la sola utilización de estos signos podría llevar a un subdiagnóstico debido a que la precisión diagnóstica es solo moderada (LR no menores a 0,1 ni mayores a 10). Esto, sumado al poco impacto que estas intervenciones han tenido en la mortalidad infantil por neumonía (11), hace urgente la realización de estudios con adecuada calidad metodológica, que permitan aclarar el valor real de estos y otros signos clínicos en el diagnóstico y manejo de la NAC en niños menores de cinco años.

Nuestra revisión tiene limitaciones propias de este tipo de estudio que incluye la posibilidad no descartable de sesgo de publicación que intentamos reducir contactando expertos en el tema realizando una búsqueda exhaustiva. Esta búsqueda incluyó cuatro bases de datos principales, dos de las cuales coleccionan evidencia producida en países con bajos ingresos. Además, agregamos a la búsqueda electrónica una manual. La selección de estudios fue reproducible así como la asignación de criterios de calidad metodológica. No pudimos explorar los subgrupos considerados para explicar la inconsistencia en los resultados dado el número reducido de estudios elegibles.

Como se mencionó en la sección de calidad metodológica, los estudios encontrados tienen problemas metodológicos que potencialmente afectan nuestros resultados debido a que se basan en estudios con sesgos de selección, que presentaron pérdidas en el seguimiento y que probablemente miden diferentes espectros de la NAC.

En conclusión, de manera especial en países con bajos recursos, en donde la disponibilidad de médicos no es universal y un grupo importante de pacientes es atendido por personal técnico, los trabajadores sanitarios (médicos o no) podrían usar la presencia o ausencia de taquipnea y retracciones subcostales como ayuda en la evaluación de un paciente en el que se sospeche $\mathrm{NAC}$, incluso a pesar de la incertidumbre relativa en su poder diagnóstico y las proporciones de posibilidad relativamente modesta.

Esta recomendación es débil, debido a la baja calidad de evidencia que la sustenta. Esto obliga a realizar estudios con una mejor calidad metodológica que evalúen la precisión y exactitud de taquipnea, retracciones subcostales y otros signos clínicos. Estos estudios deberían seguir un diseño de ensayo clínico aleatorizado, considerando los criterios clínicos de la OMS como una intervención, y compararlos contra un esquema diagnóstico convencional con el fin de disminuir un posible sesgo de selección e incrementar la aplicabilidad de sus resultados. Estos estudios deben de ir más allá que la simple detección de NAC y evaluar resultados importantes para los pacientes como mortalidad infantil debido a neumonía, hospitalización en cuidados intensivos, necesidad de intubación, ventilación mecánica y análisis costo beneficio.

Contribuciones de autoría: JPD, GP, MAL, CS, VM, GM participaron en la planificación, conducción y posterior elaboración del presente manuscrito; PE participo en la elaboración y conducción de la estrategia de búsqueda electrónica, LH y YP participaron en la realización del protocolo así como también en la estrategia de búsqueda manual.

Fuentes de financiamiento: autofinanciado.

Conflictos de interés: todos los autores declaran no tener ningún conflicto de interés.

\section{REFERENCIAS BIBLIOGRÁFICAS}

\section{REFERENCIAS BIBLIOGRÁFICAS}

1. Harris M, Clark J, Coote N, Fletcher P, Harnden A, McKean M, et al. British Thoracic Society guidelines for the management of community acquired pneumonia in children: update 2011. Thorax. 2011;66 Suppl 2:ii1-23.
2. Organización Mundial de la Salud. Neumonia [Internet]. Centro de prensa. Nota descriptiva $\mathrm{N}^{\circ} 331$. Ginebra: OMS; 2011 [citado 9 abril 2012]. Disponible en http://www.who. int/mediacentre/factsheets/fs331/es/ index.html
3. Rudan I, Boschi-Pinto C, Biloglav Z, Mulholland $\mathrm{K}$, Campbell $\mathrm{H}$. Epidemiology and etiology of childhood pneumonia. Bull World Health Organ. 2008;86(5):408-16.

4. United Nations Children's Fund. Levels \& trends in child mortality. 
Report 2011. New York: The Fund; 2011.

5. Sazawal S, Black RE; Pneumonia Case Management Trials Group. Effect of pneumonia case management on mortality in neonates, infants, and preschool children: a meta-analysis of community-based trials. Lancet Infect Dis. 2003;3(9):547-56.

6. Theodoratou E, Al-Jilaihawi S, Woodward F, Ferguson J, Jhass A, Balliet $\mathrm{M}$, et al. The effect of case management on childhood pneumonia mortality in developing countries. Int J Epidemiol. 2010;39 Suppl 1:i155-71.

7. Marsh DR, Gilroy KE, Van de Weerdt R, Wansi E, Qazi S. Community case management of pneumonia: at a tipping point? Bull World Health Organ. 2008;86(5):381-9.

8. Organización Mundial de la Salud. Technical bases for the WHO recommendations on the management of pneumonia in children at first-level health facilities. Geneva: OMS; 1991.

9. Leventhal JM. Clinical predictors of pneumonia as a guide to ordering chest roentgenograms. Clin Pediatr (Phila). 1982;21(12):730-4.

10. Shann F, Hart K, Thomas D. Acute lower respiratory tract infections in children: possible criteria for selection of patients for antibiotic therapy and hospital admission. 1984. Bull World Health Organ. 2003;81(4):301-5.

11. Mulholland K. Childhood pneumonia mortality--a permanent global emergency. Lancet. 2007;370(9583):285-9.

12. Whiting P, Rutjes AW, Reitsma JB, Bossuyt PM,KleijnenJ.The development of QUADAS: a tool for the quality assessment of studies of diagnostic accuracy included in systematic reviews. BMC Med Res Methodol. 2003;3:25.

13. Zamora J, Abraira V, Muriel A, Khan K, Coomarasamy A.Meta-DiSc: a software for meta-analysis of test accuracy data. BMC Med Res Methodol. 2006;6:31.

14. Glas AS, Lijmer JG, Prins MH, Bonsel GJ, Bossuyt PM. The diagnostic odds ratio: a single indicator of test performance. J Clin Epidemiol. 2003;56(11):1129-35.

15. Higgins JP, Thompson SG, Deeks JJ, Altman DG. Measuring inconsistency in meta-analyses. BMJ. 2003;327(7414):557-60.

16. World Development Indicators [Internet]. Washington, DC: The World Bank; 2010. [citado el 17 de febrero del 2012]. Disponible en: http://data.worldbank.org/datacatalog/world-development-indicators

17. Dai Y, Foy HM, Zhu Z, Chen B, Tong F. Respiratory rate and signs in roentgenographically confirmed pneumonia among children in China. Pediatr Infect Dis J. 1995;14(1):48-50.

18. Falade AG, Tschappeler H, Greenwood BM, Mulholland EK. Use of simple clinical signs to predict pneumonia in young Gambian children: the influence of malnutrition. Bull World Health Organ. 1995;73(3):299-304.

19. Harari M, Shann F, Spooner V, Meisner S, Carney M, de Campo J. Clinical signs of pneumonia in children. Lancet. 1991;338(8772):928-30.

20. Palafox $M$, Guiscafré $H$, Reyes $H$, Munoz O, Martinez H. Diagnostic value of tachypnoea in pneumonia defined radiologically. Arch Dis Child. 2000;82(1):41-5.

21. Redd SC, Vreuls R, Metsing M, Mohobane PH, Patrick E, Moteetee M. Clinical signs of pneumonia in children attending a hospital outpatient department in Lesotho. Bull World Health Organ. 1994;72(1):113-8.

22. Shamo'on $H$, Hawamdah A, Haddadin $\mathrm{R}$, Jmeian $\mathrm{S}$. Detection of pneumonia among children under six years by clinical evaluation. East Mediterr Health J. 2004;10(4-5):482-7.

23. Shah S, Bachur R, Kim D, Neuman MI Lack of predictive value of tachypnea in the diagnosis of pneumonia in children. Pediatr Infect Dis J. 2010;29(5):406-9

24. Singhi S, Dhawan A, Kataria S, Walia BN. Clinical signs of pneumonia in infants under 2 months. Arch Dis Child. 1994;70(5):413-7

25. Landis JR, Koch GG. The measurement of observer agreement for categorical data. Biometrics. 1977;33(1):159-74.

26. Montori VM, Wyer P, Newman TB, Keitz S, Guyatt G; EvidenceBased Medicine Teaching Tips Working Group. Tips for learners of evidence-based medicine: 5. The effect of spectrum of disease on the performance of diagnostic tests. CMAJ. 2005;173(4):385-90.

27. Margolis P, Gadomski A. Does this infant have pneumonia? JAMA. 1998;279(4):308-13.

28. Zukin DD, Hoffman JR, Cleveland RH, Kushner DC, Herman TE. Correlation of pulmonary signs and symptoms with chest radiographs in the pediatric age group. Ann Emerg Med. 1986;15(7):792-6.

29. Schünemann HJ, Oxman AD, Brozek J, Glasziou P, Jaeschke R, Vist GE, et al. Grading quality of evidence and strength of recommendations for diagnostic tests and strategies. BMJ. 2008;336(7653):1106-10.

Correspondencia: Germán Málaga

Dirección: Calle Chavin 159. CCMonterrico.

Lima 33, Perú.

Teléfono: (51) 992768300

Correo electrónico:german.malaga@upch.pe 